\title{
Conceptual combination theory and technological imagination cognition:
}

technological imagination test construction

Yung-Chieh Yen ${ }^{1}$ Ching-Wen Wang ${ }^{2}$ Ruey-Yun Horng ${ }^{3}$

\section{Introduction}

Imagination is the basic of creativity, and it is also the motivity of human thinking development. By unlimitedly imagining, people innovate new concept or product. Therefore, imagination is called the ground of creation (Ho et al., 2013). Imagination and creativity are also key elements to drive social economy and culture (Wang et al., 2015; McCormack, 2010). What's more, there are lots of connection within people's thinking, language, and life experience ( Wang et al, 2015;Mountain, 2007;Adams, 2004; Grant, 2004). In the few years, there were many studies about imagination, and some of them had started to take research on different types of it, such as Technological Imagination (Wang, Ho, \& Cheng, 2015; Wang, Ho, Wu, \& Cheng, 2014; Liang \& Chang, 2014; Ho, Wang, \& Cheng, 2013; Yueh, Chang, \& Liang, 2013), News Imagination (Liao, Chang, Liang, \& Liang, 2016), Scientific Imagination process (Magid, Sheskin, \& Schulz, 2015; Wang et al., 2015 ;Ho et al., 2013), Creative Imagination (Chiu, 2012) and so on.

In the study of Art Education Partnership 2008, it pointed out that there were $89 \%$ of responders who thought creative thinking played an important role in self-innovative ability in the economic environment of 21st century. Under the fast-changing world now, people who have talent of imagination and innovation are popular to business. Thus, how to hire an imaginative person and how to inspire employee's imagination and creativity becomes the essential elements for business to heave their competition. However, we are weak on the understanding of imagination and talents cultivation.

The purpose of this study was to explain the imagination thinking process by Conceptual Combination Theory, to produce a measure tool to test Technological Imagination, to examine its reliability and validity, and to set up evaluation norm of Taiwan undergraduate.

\section{Test Construction}

The major subjects in this study were undergraduates and graduates in Taiwan.

Retrieved Tests : 802

Validated Tests : 714

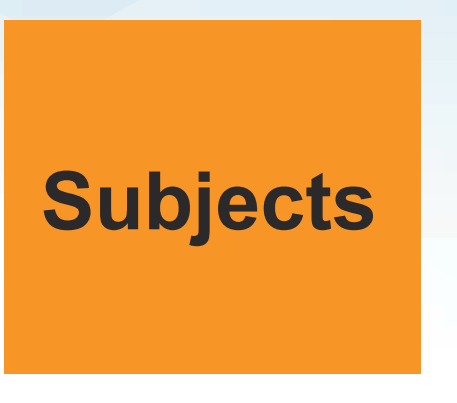

Department of Business Management, National Chung Hsing University, Taichung, Taiwan , ajyean@ms9.hine

2 Department of Business Management, National Chung Hsing University, Taichung, Taiwan

${ }^{3}$ Department of Industrial Engineering and Management, National Chiao Tung University, Hsinchu, Taiwan

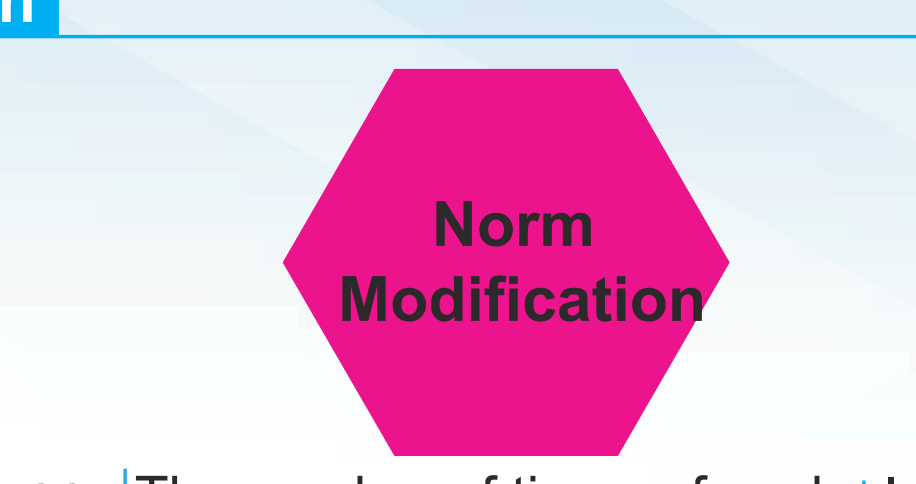

The number of times of each ed, and the number of reactions was $5 \%$ or more, and the number of reactions was $5 \%$ or less, but the class of

\section{Evaluate} ror Processing

idea had to use both concepts at the same time If the description only took one of the nouns or the description is not reasonable, it is 0 .

At the end, calculated six reactions' grade to be its total score. type of reaction was count$2 \%$ or more was counted as 1 point, and a scoring norm was produced.

\section{Reliability and validity analysis}

\section{Item Analysist}

The total score was added up by the test of Technological Imagination from 714 subjects. The correlation between each question is .010 .144, mostly it was not obvious; the correlation between each question's score and the total score is .376 .543, all of that reached .01 level of significance

\section{Discrimination Analysis}

The total score was added up by the test of Technological Imagination from 714 subjects. The score of top 27\%,246 subjects, was high score type $(M=7.37, S D=1.416$,

rande $=6 \sim 12$ ). The score of last $27 \%, 249$ subjects, is low score type $(M=1.92, S D=1.069$, rande $=0 \sim 3)$ with an independent sample $t$ to compare whether it had disparity between each score in the question or not. As a result, each of the questions was between 9.845 16.736 ( $p$ 's <.1) each middle high type subjects were apparently higher than low score type. It showed that discrimination was good on each question.

\section{Reliability Snalysis}

Retrieved a total of 32 graduates' tests. At the concept of the imagination, the test-retest reliability after a week was $0.495(p<.01)$, it showed it was well stability on the section.
14

80

60

40

0 Technological Imagination were between 0 to 12 $(\mathrm{M}=4.58, \mathrm{SD}=2.51)$. It was normal distribution.

\section{Scorer Reliability}

In this study, the scorer reliability was done by two scorers who had score training in advance. Scorers graded its conceptual combination, and its reliability was $.723(p<.001)$. It showed the scorer reliability was good.

Criterion-Related Validity

It targeted 145 undergraduates who majored in design as subjects, and based on the mid-term exam of design course as criterion-related validity, the correlation coefficient was $.475(p<.001)$ which showed the criterion-related validity of this test was good.

\section{Conclusion and discussion}

Conceptual Combination Theory gives technological imag ination a cognitive explanation. The test of technological imagination based on Conceptual Combination Theory can not only be a tool for education institute to understand student's imagination, but also can be materials to promote creativity related courses or activities. Nowadays, the needs of creative talents get higher and higher in Taiwan's industry. The test of technological imagination offers a ready-made too for human resources to hire creative talents, and Conceptual Combination Theory offers a training material to inspire imagination and promote innovation ability which is helpful for business to conduct creativity training. 